Pacific Journal of Mathematics

WITT KERNELS OF FUNCTION FIELD EXTENSIONS 


\title{
WITT KERNELS OF FUNCTION FIELD EXTENSIONS
}

\author{
ROBERT W. FitzGerald
}

\begin{abstract}
Let $F$ be a field of characteristic not 2. For a non-hyperbolic quadratic form $q$ of dimension at least 2 , let $F(q)$ denote the function field of the projective variety $q=0$. We consider the problem, explicitly raised as problem $D$ by Lam, of determining the kernel of induced map of Witt rings $W F \rightarrow W F(q)$. This kernel is the Witt kernel of the field extension and is denoted by $W(F(q) / F)$. The basic tool is a comparison of $W(F(q \perp\langle x\rangle) / F)$ and $W(F(q) / F)$. The Witt kernels $W(F(q) / F)$ where $q$ has small dimension or $F$ has small Hasse number are determined. Applications are made to the question of when a conservative form is embeddable.
\end{abstract}

In the case $q$ is a Pfister form, the function fields $F(q)$ have been widely used (e.g. the Arason-Pfister Hauptsatz). Central to the applications is that the Witt kernel $W(F(q) / F)$ is $q W F$ for Pfister forms $q$. Elman, Lam and Wadsworth have considered function fields of several Pfister forms $\rho_{i}$, (cf. [8]). Again the basic problem is computing the Witt kernel $W\left(F\left(\rho_{1}, \rho_{2}, \ldots, \rho_{r}\right) / F\right)$ and showing it is a Pfister ideal.

Here also the emphasis is on finding conditions to insure Witt kernels are generated by Pfister forms. In the first section the comparison of $W(F(\varphi \perp\langle x\rangle) / F)$ and $W(F(\varphi) / F)$ is made and this is applied in the second section to forms of small dimension. For example, we show the Witt kernel $W(F(\varphi) / F)$ is a strong Pfister ideal if $\varphi$ has dimension $\leq 5$ and a Pfister ideal if dimension 6 . This is used to improve several results of Gentile and Shaprio (in [12]) on their question of when $W(F(\varphi) / F$ ) contains a non-zero Pfister form.

The last section treats fields $F$ of finite Hasse number. It is shown that all Witt kernels of function fields are strong Pfister ideals if $\tilde{u}(F) \leq 8$. And the Witt kernels $W(F(\varphi) / F)$ are essentially computed for any form $\varphi$ over $F$ with $\tilde{u}(F) \leq 32$. Examples of fields with Hasse number $\leq 8$ are $C_{3}$ fields, global and local fields, and finite fields.

The notation and terminology used are basically those of [15]. Isometry of forms $\alpha$ and $\beta$ are denoted by $\alpha \simeq \beta$, while equality in the Witt ring is written $\alpha=\beta$. The uniquely determined maximal anisotropic subform $\alpha$ of a form $\beta$ is termed the kernel of $\beta$ and written as $\alpha=\operatorname{ker}(\beta)$. If $x \alpha \simeq \beta$ for some $x \in \dot{F}$, we say $\alpha$ and $\beta$ are similar. The $u$-invariant used in the 
last two sections is the generalized $u$-invariant of Elman and Lam (see e.g. [4]) and not the one discussed in [15].

The set of all $F$-Pfister forms is denoted by $P(F)$ and $P_{n}(F)$ denotes the set of $n$-fold $F$-Pfister forms. The set of forms over $F$ similar to $F$-Pfister forms [ $n$-fold $F$-Pfister forms] is denoted by $G P(F)$ [resp. $\left.G P_{n}(F)\right]$. If $\rho \in G P(F)$ is anisotropic and $\varphi<\rho$ then $\varphi$ is a Pfister neighbor if $2 \operatorname{dim} \varphi>\operatorname{dim} \rho$ and a conjugate neighbor if $2 \operatorname{dim} \varphi=\operatorname{dim} \rho$.

We use the terms conservative and embeddable forms as defined by Gentile and Shapiro. Namely, a form $q$ is conservative if $W(F(q) / F) \neq 0$, or equivalently, if $q \otimes L$ is anisotropic for every field extension $L / F$ with $W(L / F)=0$. A form $q$ is embeddable if it is similar to a subform of an anisotropic Pfister form.

Following Elman, Lam and Wadsworth, for a subset $N \subset \mathbf{N}$ and $\mathfrak{A}$ an ideal of $W F$ we say $\mathfrak{A}$ is an $\mathbf{N}$-Pfister ideal of $\mathfrak{A}$ is generated by $r$-fold Pfister forms, $r \in N$. $\mathfrak{A}$ is a strong $\mathbf{N}$-Pfister ideal if each $q \in \mathfrak{U}$ is isometric to a sum of scalar multiples of $r$-fold Pfister forms in $\mathfrak{A}, r \in N$. We write $n$-Pfister for $\{n\}$-Pfister.

Let $X_{F}$ denote the set of orderings on the field $F$ and topologize $X_{F}$ by taking as an open subbasis the Harrison sets:

$$
H_{F}(a)=\left\{\alpha \in X \mid a>_{\alpha} 0\right\},
$$

where $a$ ranges over $\dot{F}$. A form $q$ is indefinite at $\alpha \in X_{F}$ if $\left|\operatorname{sgn}_{\alpha} q\right|<\operatorname{dim} q$ and indefinite if $q$ is indefinite at all $\alpha \in X_{F}$. The Hasse number of $F$ is:

$$
\tilde{u}(F)=\max \{\operatorname{dim} q \mid q \text { anisotropic and indefinite over } F\}
$$

if the maximum exists, otherwise $\tilde{u}(F)=\infty$.

Knebusch's important paper [13] will be used extensively and notation and terminology not found in [15] or mentioned above will be taken from it. In particular, we use the degree of a form $q$. As shown in [13], for $q \neq 0$ the $\min \{\operatorname{dim}(\operatorname{ker}(q \otimes K)) \mid K / F$ such that $q \otimes K \neq 0\}$ is a 2-power $2^{d}$. The degree of $q$ is $d$ (if $q=0$, the degree of $q$ is $\infty$ ). We also use the ideal $J_{n} F=\{q \in W F \mid \operatorname{deg} q \geq n\}$.

1. Witt kernels and strong Pfister ideals. The following basic results will be used frequently:

(a) If $\varphi$ is a neighbor to the $n$-fold Pfister form $\rho$, then $W(F(\varphi) / F)$ is a strong $n$-Pfister ideal $([5,1.4])$.

(b) (Cassels-Pfister theorem.) Let $q$ and $\varphi$ be anisotropic forms such that $q \otimes F(\varphi)=0$. Then for each $x \in D(q) \cdot D(\varphi)$, there exists a form $\eta_{x}$ over $F$ such that $x q \simeq \varphi \perp \eta_{x}$. 
LEMMA 1.1. Suppose $\psi$ is a subform of a form $\varphi$. Then $W(F(\varphi) / F) \subset$ $W(F(\psi) / F)$.

Proof. Since $\varphi \otimes F(\psi)$ is isotropic, there is an $F$-place $F(\varphi) \rightarrow F(\psi)$ $\cup \infty$ and so $W(F(\varphi) / F) \subset W(F(\psi) / F)$ (cf. [13]).

We begin the computations:

Proposition 1.2. Let $\varphi$ and $\psi$ be anisotropic forms over $F$ with $1 \in D(\psi)$ and $\varphi \simeq \psi \perp\langle x\rangle$, for some $x \in \dot{F}$. If $W(F(\psi) / F)$ is a strong n-Pfister ideal then:

(i) $W(F(\varphi) / F)$ is a $\{n, n+1\}$-Pfister ideal.

(ii) If $\sigma \in W(F(\varphi) / F) \cap P_{k}(F)$, with $k \geq n+1$, then there is a $\rho \in W(F(\varphi) / F) \cap P_{n+1}(F)$ such that $\rho \mid \sigma$.

Proof. (i) We have $W(F(\varphi) / F) \subset W(F(\psi) / F$ ) by (1.1). Let $q \in$ $W(F(\varphi) / F)$ be anisotropic; we may assume $1 \in D(q)$. Now $q \in$ $W(F(\psi) / F)$, a strong $n$-Pfister ideal, so we may write:

$$
q \simeq c_{1} \rho_{1} \perp \cdots \perp c_{k} \rho_{k}
$$

where $c_{i} \in \dot{F}$ and $\rho_{l} \in W(F(\psi) / F) \cap P_{n}(F)$. We use induction on $k$ to show $q$ equals a sum of multiples of $n$-fold and $(n+1)$-fold Pfister forms in $W(F(\varphi) / F)$. The case $k=1$ is trivial, so suppose $k>1$.

Since $1 \in D(q)$, we may assume $c_{1}=1$, by [10, 3.1]. By the CasselsPfister theorem, as $1 \in D(q) \cap D(\varphi), 1 \in D(\psi) \cap D\left(\rho_{1}\right)$, we have:

$$
\begin{gathered}
q \simeq \varphi \perp q_{1} \simeq \psi \perp\langle x\rangle \perp q_{1} \\
\rho_{1} \simeq \psi \perp \gamma
\end{gathered}
$$

for some forms $q_{1}$ and $\gamma$ over $F$. Cancelling $\psi$ from the isometry (*) yields

$$
x \in D\left(\gamma \perp \frac{k}{\perp=2} c_{i} \rho_{i}\right) \text {. }
$$

Thus $x=a+b$, with

$$
a \in D(\gamma) \cup\{0\}, b \in D\left(\frac{k}{\perp} c_{i} \rho_{i}\right) \cup\{0\} .
$$

Case 1. $b=0$.

Here $x \in D(\gamma)$ and so $\varphi \simeq \psi \perp\langle x\rangle<\rho_{1}$. Hence $\rho_{1} \in W(F(\varphi) / F)$ and $\perp_{i \neq 2}^{k} c_{i} \rho_{l} \in W(F(\varphi) / F)$. By induction $\perp_{i=2}^{k} c_{i} \rho_{i}$ equals a sum of multiples of $n$-fold and $(n+1)$-fold Pfister forms in $W(F(\varphi) / F)$. Thus so is $q \simeq \rho_{1} \perp \perp_{i=2}^{k} c_{i} \rho_{i}$. 
Case 2. $b \neq 0$.

Here we may assume $c_{2}=b$ by [10, 3.10]. Since $x \in D(\gamma \perp\langle b\rangle)$, $\varphi \simeq \psi \perp\langle x\rangle\left\langle\rho_{1} \perp\langle b\rangle\left\langle\rho_{1} \otimes\langle 1, b\rangle\right.\right.$. Now, since $W(F(\psi) / F)$ is a strong $n$-Pfister ideal, $\rho_{1}$ and $\rho_{2}$ are linked $([10,3.1])$. Say $\rho_{i}=\mu \otimes\left\langle 1, y_{i}\right\rangle$ $(i=1,2)$, where $\mu \in P_{n-1}(F)$ and $y_{1}, y_{2} \in \dot{F}$. Then:

$$
\begin{aligned}
\rho_{1} \perp b \rho_{2} & =\mu \otimes\left\langle 1, y_{1}, b, b y_{2}\right\rangle \\
& =\mu \otimes\left\langle\left\langle y_{1}, b\right\rangle\right\rangle \perp b y_{2} \mu \otimes\left\langle 1,-y_{1} y_{2}\right\rangle .
\end{aligned}
$$

Note $\mu \otimes\left\langle\left\langle y_{1}, b\right\rangle\right\rangle \simeq \rho_{1} \otimes\langle 1, b\rangle \in W(F(\varphi) / F)$, since it contains $\varphi$ as a subform. So:

$$
q \perp-\mu \otimes\left\langle\left\langle y_{1}, b\right\rangle\right\rangle=b y_{2} \mu \otimes\left\langle 1,-y_{1} y_{2}\right\rangle \perp \frac{k}{\perp} c_{i} \rho_{i} \in W(F(\varphi) / F) .
$$

The left hand side is also in $W(F(\psi) / F)$, a strong $n$-Pfister ideal, and thus its kernel is isometric to a sum of multiples of at most $k-1 n$-fold Pfister forms in $W(F(\psi) / F)$. Thus by induction, $q \perp-\mu\left\langle\left\langle y_{1}, b\right\rangle\right\rangle$, and hence $q$ is a sum of multiples of $n$-fold and $(n+1)$-fold Pfister forms in $W(F(\varphi) / F)$.

(ii) Repeat the argument in (i), with $\sigma$ replacing $q$. In Case 1, $\rho_{1} \in W(F(\varphi) / F) \cap P_{n}(F)$ and $\rho_{1}$ is a subform of $\sigma$. Hence $\rho_{1} \mid \sigma$, by [5, 2.7]. So take any form $\rho \in W(F(\varphi) / F) \cap P_{n+1}(F)$ such that $\rho_{1} \mid \rho$ and $\rho \mid \sigma$.

In Case 2, let $\rho=\rho_{1} \otimes\langle 1, b\rangle$. We know $\rho_{1} \perp\langle b\rangle$ is a neighbor of $\rho$ and a subform of $\sigma$. Thus $F(\rho) \sim_{F} F\left(\rho_{1} \perp\langle b\rangle\right)$ and $\sigma \otimes F\left(\rho_{1} \perp\langle b\rangle\right)=0$, by [13, 4.1]. So $\sigma \otimes F(\rho)=0$ and $\rho \mid \sigma$ by $[5,1.4]$. Also, the argument in (i) showed $\varphi<\rho_{1} \perp\langle b\rangle$, so $\varphi<\rho$ and thus $\rho \in W(F(\varphi) / F) \cap P_{n+1}(F)$.

Corollary 1.3. Suppose $\varphi \simeq \psi \perp\langle x\rangle$ with $x \in \dot{F}$ and $W(F(\psi) / F) a$ strong $(n-1)$-Pfister ideal. If $W(F(\varphi) / F) \cap P_{n-1}(F)=0$ and $W(F(\varphi) / F)$ is n-linked, then $W(F(\varphi) / F)$ is a strong $n$-Pfister ideal.

Proof. $W(F(\varphi) / F) \cap P_{n-1}(F)=0$ and (1.2)(i) imply $W(F(\varphi) / F)$ is an $n$-Pfister ideal. Then (1.2)(ii) and the hypothesis on linkage imply the result by $[10,3.1]$.

Proposition 1.4. Let $\psi$ be a neighbor of $\rho \in P_{n}(F)$ and let $\varphi \simeq \psi \perp$ $\langle x\rangle$, with $x \in \dot{F}$, be anisotropic. Then either:

(a) $\varphi$ is a neighbor of $\rho$ and $W(F(\varphi) / F)=\rho W F$, a strong $n$-Pfister ideal, or

(b) $\varphi$ is not a neighbor of $\rho$ and $W(F(\varphi) / F)$ is a strong $(n+1)$-Pfister ideal. 
Proof. We need only show (b) so assume $\varphi$ is not a neighbor of $\rho$. By scaling if necessary, we may assume $\psi$, and hence $\varphi$, represent 1 .

$W(F(\psi) / F)=\rho W F$ is a strong $n$-Pfister ideal. We wish to apply (1.3). Suppose $0 \neq \sigma \in W(F(\varphi) / F) \cap P_{n}(F)$. Since $1 \in D(\varphi) \cap D(\sigma)$, the Cassels-Pfister theorem implies that $\varphi$ is a subform of $\sigma$ and hence so is $\psi$. Since $\psi$ is a neighbor of the $n$-fold Pfister form $\rho, \operatorname{dim} \psi>2^{n-1}$. Thus $\psi$ is a neighbor of $\sigma$ and $\rho \simeq \sigma([\mathbf{1 3}, 7.4])$. Thus $\varphi$ is a neighbor of $\rho$. Contradiction.

Thus $W(F(\varphi) / F) \cap P_{n}(F)=0$. Since $W(F(\varphi) / F) \subset W(F(\psi) / F)$ $=\rho W F$, any two $(n+1)$-fold Pfister forms in $W(F(\varphi) / F)$ are linked by $\rho$. So the result follows from (1.3).

COROLlaRY 1.5. Let $\varphi$ be an anisotropic form such that $\operatorname{dim} \varphi=4$ and $\varphi \notin G P(F)$. If $W(F(\varphi) / F) \neq 0$, then $W(F(\varphi) / F)$ is a strong 3-Pfister ideal. In particular, $\varphi$ is conservative if and only if $\varphi$ is a conjugate neighbor.

Proof. By scaling we may assume $\varphi \simeq\langle 1, a, b, x\rangle$, for some $a, b$, $x \in \dot{F}$. The first statement then follows from (1.4) and the second from the Cassels-Pfister theorem.

\section{2. $W(F(\varphi) / F)$ for small dimensional $\varphi$.}

Remark. Let $\rho$ be an $n$-fold Pfister form over $F$. Suppose $\rho \simeq \psi \perp \gamma$, with $\operatorname{dim} \psi>\operatorname{dim} \gamma$, and $\varphi \simeq \psi \perp\langle x\rangle$ is anistropic. Further suppose $\varphi$ is not a neighbor of $\rho$. Then, $W(F(\varphi) / F)$ is a strong $(n+1)$-Pfister ideal by (1.4). By examining the proof of (1.2) we see that:

$$
P_{n+1}(F) \cap W(F(\varphi) / F)=\{\rho \otimes\langle 1, a\rangle \mid a \in D(\langle x\rangle \perp-\gamma)\} .
$$

Now $W(F(\psi) / F)=\rho W F$ and:

$$
\rho W F \cap\langle 1, x\rangle W F \cap P_{n+1}(F)=\left\{\rho \otimes\langle 1, a\rangle \mid a \in D\left(\langle x\rangle \perp-\rho^{\prime}\right)\right\},
$$

where $\rho^{\prime}$ is the pure part of $\rho$. Thus:

$$
W(F(\varphi) / F) \subset W(F(\psi) / F) \cap\langle 1, x\rangle W F,
$$

but the inclusion may be strict. 
We wish to examine in detail the structure of $W(F(\varphi) / F$ ) for four dimensional forms:

EXAMPLE. Let $\varphi$ be conservative and $\operatorname{dim} \varphi=4$; we may assume $\varphi \simeq\langle 1, a, b, x\rangle$. Suppose $x \neq a b$. Then:

$$
\begin{aligned}
W(F(\varphi) / F) \cap P_{3}(F) & =\{\langle\langle a, b, \alpha\rangle\rangle \mid \alpha \in D(\langle x,-a b\rangle)\} \\
& =\left\{\left\langle\left\langle a, b, x t^{2}-a b s^{2}\right\rangle\right\rangle \mid s, t \in F\right\} .
\end{aligned}
$$

If $t=0$, then $\left\langle\left\langle a, b, x t^{2}-a b s^{2}\right\rangle\right\rangle \simeq\langle\langle a, b,-a b\rangle\rangle=0$. So we may assume $t \neq 0$. Hence:

$$
W(F(\varphi) / F) \cap P_{3}(F)=\left\{\left\langle\left\langle a, b, x-a b s^{2}\right\rangle\right\rangle \mid s \in F\right\} \cup\{0\} .
$$

In particular:

$$
W(F(\varphi) / F)=\langle\langle a, b\rangle\rangle \sum_{s \in F}\left\langle\left\langle x-a b s^{2}\right\rangle\right\rangle W F .
$$

by (1.5).

Comparing with (1.5), we also have:

$\varphi$ is conservative iff $\varphi$ is a conjugate neighbor

$$
\text { iff } D(\langle-x, a b\rangle) \not \subset D(\langle\langle a, b\rangle\rangle) \text {. }
$$

To treat 5 and 6 dimensional forms, we need:

THEOREM 2.1. Let $\psi$ be a codimension 1 neighbor of $\rho \in P_{n}(F)$, $\varphi \simeq \psi \perp\langle x, y\rangle$ anisotropic and suppose $\varphi$ is not a Pfister neighbor. Then $W(F(\varphi) / F)$ is a strong $(n+2)$-Pfister ideal.

Proof. By (1.4) $W(F(\psi \perp\langle x\rangle) / F)$ is a strong $(n+1)$-Pfister ideal, and so $W(F(\varphi) / F)$ is a $\{n+1, n+2\}$-Pfister ideal, by (1.2). Since $\operatorname{dim} \varphi=2^{n}+1$ and $\varphi$ is not a Pfister neighbor $W(F(\varphi) / F) \cap P_{n+1}(F)$ $=0$. Thus, by (1.3), we need only show $W(F(\varphi) / F)$ is $(n+2)$-linked.

Let $\rho_{1}, \rho_{2} \in W(F(\varphi) / F) \cap P_{n+2}(F)$. By the Cassels-Pfister theorem, $\varphi$ is similar to a subform of each $\rho_{i}$ and so the Witt index $i\left(\rho_{1} \perp-\rho_{2}\right) \geq$ $2^{n}+1$. But $i\left(\rho_{1} \perp-\rho_{2}\right)$ must be a power of 2 , by $[5,4.5]$. Thus $i\left(\rho_{1} \perp-\rho_{2}\right) \geq 2^{n+1}$ and hence $\rho_{1}$ and $\rho_{2}$ are linked.

COROllary 2.2. Let $\varphi$ be a conservative form of dimension 5. Then either:

(a) $\varphi$ is a neighbor to a Pfister form $\rho$ and $W(F(\varphi) / F)=\rho W F$ is a strong 3-Pfister ideal, or

(b) $\varphi$ is not a Pfister neighbor and $W(F(\varphi) / F)$ is a strong 4-Pfister ideal. 
It is quite possible that a 5 dimensional form $\varphi$ is not a Pfister neighbor. Indeed $\varphi$ is a Pfister neighbor if and only if $d(\varphi) \in D(\varphi)$, by $[13$, p. 10].

COROLlaRy 2.3. Let $\varphi$ be a conservative form of dimension 6. If $\varphi$ is not a Pfister neighbor then $W(F(\varphi) / F)$ is a $\{4,5\}$-Pfister ideal.

EXAMPLE. If $\varphi$ has dimension $6, W(F(\varphi) / F)$ need not be a strong Pfister ideal. Since no such example is in the literature, I will work out one in some (but not complete) detail.

Let $F=\mathbf{R}(x, y, z), \varphi=\langle 1,1,1, x, y, z\rangle, \rho_{1}=\langle\langle 1,1, x, y, z\rangle\rangle$ and $\rho_{2}=\langle\langle 1,1, x, y-1, z-x\rangle\rangle$. By considering an ordering for which $\left.z\right\rangle$ $y>x>1$, one sees that $\rho_{1}$ and $\rho_{2}$ are anisotropic. A simple computation shows $\varphi<\rho_{1}$ and $\varphi<\rho_{2}$, while a more tedious one shows $\rho_{1}$ and $\rho_{2}$ are not linked. Let $\psi=\operatorname{ker}\left(\rho_{1} \perp-\rho_{2}\right) \in W(F(\varphi) / F)$.

Fix an ordering $\alpha$ on $F$ with $x$ infinitely large positive, $y$ infinitely small positive and $z$ infinitely larger than $x$.

Claim. There does not exist $\sigma \in W(F(\varphi) / F) \cap P_{4}(F)$ such that $\operatorname{sgn}_{\alpha} \sigma=16$.

We first note that $\langle 1,1,1, x, y\rangle$ is not a Pfister neighbor - otherwise $x y \in D(\langle 1,1,1, x, y\rangle)$ and $\langle 1,1,1, x\rangle \perp y\langle 1,-x\rangle$ is isotropic, which is impossible. Thus if there is a $\sigma$ invalidating the claim, the proof of (1.2) shows we may write $\sigma \simeq\left\langle\left\langle 1,1, p^{2} x-q^{2}, r^{2} y-\beta\right\rangle\right\rangle$, where $p, q, r, \beta \in$ $\mathbf{R}[x, y, z]$ and $\beta \in\left(p^{2} x-q^{2}\right) D(\langle 1,1,1, x\rangle)$.

We will show $z \notin D(\sigma)$ and hence $\varphi \nless \sigma$. We need some simple calculations. For a polynomial $g(x, y, z) \in \mathbf{R}[x, y, z]$ let $\operatorname{deg}_{x} g$ denote the degree of $g$ as a polynomial in $x$ over $\mathbf{R}[y, z]$. Define $\operatorname{deg}_{y} g$ and $\operatorname{deg}_{z} g$ similarly.

Consider $p^{2} x, q^{2}$ and $r^{2} y$ as polynomials in $z$ over $\mathbf{R}[x, y]$, with leading coefficients $w_{1}(x, y), w_{2}(x, y)$ and $w_{3}(x, y)$ respectively. Note that $p^{2} x, q^{2}$ and $r^{2} y$ have even $z$-degree. It is easy to check the following:

(a) $\operatorname{deg}_{x} w_{1}$ is odd and $\operatorname{deg}_{x} w_{2}$ is even,

(b) $\operatorname{deg}_{y}\left(w_{1}-w_{2}\right)$ is even,

(c) $\operatorname{deg}_{y} w_{3}$ is odd.

We thus obtain:

(i) $\operatorname{deg}_{z}\left(p^{2} x-q^{2}\right)$ is even (by (a)),

(ii) $\operatorname{deg}_{z} \beta$ is even (by (i)),

(iii) $\operatorname{deg}_{z}\left(r^{2} y-\beta\right)$ is even (by (ii), (b) and (c)).

Suppose finally that $z \in D(\sigma)$. Then:

$(*) \quad z=s_{0}+\left(p^{2} x-q^{2}\right) s_{1}+\left(r^{2} y-\beta\right) s_{2}+\left(p^{2} x-q^{2}\right)\left(r^{2} y-\beta\right) s_{3}$ 
with each $s_{l}$ a sum of four squares in $F$. Let $w_{4}(x, y)$ be the $z$-leading coefficient of $\beta$. Set

$$
V=\left\{(a, b) \in \mathbf{R}^{2} \mid w_{i}(a, b)=0, \text { some } i=1,2,3, \text { or } 4\right\} ;
$$

$V$ is a closed subvariety of $\mathbf{R}^{2}$. Since $\operatorname{sgn}_{\alpha} \sigma=16, p^{2} x-q^{2}$ and $r^{2} y-\beta$ are positive with respect to $\alpha$ and we may find positive $x_{0}, y_{0} \in R-V$ such that:

$$
\left.\begin{array}{l}
P_{1}(z)=\left(p^{2} x-q^{2}\right)\left(x_{0}, y_{0}, z\right) \\
P_{2}(z)=\left(r^{2} y-\beta\right)\left(x_{0}, y_{0}, z\right)
\end{array}\right\} \geq 0 \text { for } z \gg 0 .
$$

By the observations (i) and (iii), we see that $P_{1}$ and $P_{2}$ have even degree. Thus for sufficiently negative $z_{0}$, at $\left(x_{0}, y_{0}, z_{0}\right)$ the left hand side of $(*)$ is negative while the right hand side is positive. This proves the claim.

To finish the example, suppose $W(F(\varphi) / F)$ is a strong Pfister ideal. Then we may write:

$$
\psi \approx \perp a_{i} \mu_{i}, \quad \text { with } \mu_{i} \in W(F(\varphi) / F) \cap P(F) \text { and } a_{i} \in \dot{F} \text {. }
$$

Since $\operatorname{dim} \psi=48$ we have three cases:

(i) Some $\mu_{i} \in P_{3}(F)$ :

Then $\varphi$ is a Pfister neighbor and there exists a $\sigma \in W(F(\varphi) / F) \cap P_{4}(F)$ such that $\sigma \mid \rho_{1}$. Since $\operatorname{sgn}_{\alpha} \rho_{1}=32, \operatorname{sgn}_{\alpha} \sigma=16$. Contradiction.

(ii) Some $\mu_{i} \in P_{5}(F)$ :

Then $\psi \simeq a_{1} \mu_{1} \perp a_{2} \mu_{2}$, with $\mu_{1} \in P_{5}(F)$ and $\mu_{2} \in P_{4}(F)$. But $\operatorname{deg} \psi=5$ while $\operatorname{deg}\left(a_{1} \mu_{1} \perp a_{2} \mu_{2}\right)=4$, which again is a contradiction.

(iii) All $\mu_{i} \in P_{4}(F)$ :

Then $\psi \simeq a_{1} \mu_{1} \perp a_{2} \mu_{2} \perp a_{3} \mu_{3}$, with $\mu_{i} \in W(F(\varphi) / F) \cap P_{4}(F)$. Now $\operatorname{sgn}_{\alpha} \psi=32$, as $\operatorname{sgn}_{\alpha} \rho_{1}=32$ and $\operatorname{sgn}_{\alpha} \rho_{2}=0$. Thus at least one $\mu_{i}$ has $\alpha$-signature 16, contradicting the claim.

Thus $W(F(\varphi) / F)$ is not a strong Pfister ideal.

It is worth noting that $W(F(\varphi) / F)$ does however contain 4-fold Pfister forms. For example, $0 \neq\left\langle\left\langle 1,1, x, 4 x y-(x z-x y-1)^{2}\right\rangle\right\rangle$ is in $W(F(\varphi) / F)$.

We can show $W(F(\varphi) / F)$ is a strong Pfister ideal in some cases.

COROllary 2.4. Let $\varphi$ be a conservative form of dimension 6 which is not a Pfister neighbor. If $\varphi$ contains a four dimensional subform of determinant 1 , then $W(F(\varphi) / F)$ is a strong 4-Pfister ideal. 
Proof. Write $\varphi \simeq \psi \perp\langle a, b\rangle$, with $\operatorname{dim} \psi=4$ and $d(\psi)=1$. If $c \in$ $D(\psi)$, then $c \psi \in P_{2}(F)$. So we may assume $\varphi \simeq \rho \perp\langle x, y\rangle$, where $\rho \in$ $P_{2}(F)$ and $x, y \in \dot{F}$. Now $\rho \perp\langle x\rangle$ is a neighbor to $\rho \otimes\langle 1, x\rangle$, so $W(F(\varphi) / F)$ is a strong 4-Pfister ideal by (1.4).

3. Conservative and embeddable forms. In [12], Gentile and Shapiro raised the question whether a conservative form $\varphi$ over $F$ must be embeddable. They showed the answer was yes, if $\operatorname{dim} \varphi \leq 5$ or if $u(F)<24$ ([12, Corollaries 8 and 19]). The results of Section 2 can be used to improve these bounds. As an immediate consequence of (2.3) we have:

COROllary 3.1. Let $\operatorname{dim} \varphi \leq 6$. Then $\varphi$ is conservative iff $\varphi$ is embeddable.

Proposition 3.2. Let $\varphi$ be a conservative form over $F$ which is not a Pfister neighbor and such that $\operatorname{dim} \varphi \geq 5$. Let $q \in W(F(\varphi) / F)$ be anisotropic. Then:

(a) $16 \mid \operatorname{dim} q$

(b) $q \equiv \rho \bmod I^{5} F$, where $\rho \in P_{4}(F) \cap W(F(\varphi) / F)$.

Proof. We first note that for (b) we need only show the equation holds for some $\sigma \in G P_{4}(F)$. Namely then $q=\alpha \rho \perp q_{1}$, where $\alpha \in \dot{F}, \rho \in P_{4}(F)$ and $q_{1} \in I^{5} F$. Now $\alpha \rho \otimes F(\varphi)=-q_{1} \otimes F(\varphi) \in I^{5} F(\varphi)$. By the ArasonPfister Hauptsatz ([2]), $\rho \otimes F(\varphi)=0$ and so $\rho \in W(F(\varphi) / F)$. Further $q=\rho \perp\langle-1, \alpha\rangle \rho \perp q_{1}$ and so $q \equiv \rho \bmod I^{5} F$.

Let $\psi$ be a 5-dimensional subform of $\varphi$. By (1.1), $q \in W(F(\psi) / F)$.

Case 1. $\psi$ is not a Pfister neighbor:

Here we may write $q \simeq \perp_{i=1}^{m} \alpha_{i} \sigma_{i}$, with each $\alpha_{i} \in \dot{F}$ and $\sigma_{i} \in$ $W(F(\psi) / F) \cap P_{4}(F)$, by (2.2). In particular, (a) holds. Now write:

$$
q \equiv \stackrel{\perp}{i=1}^{n} a_{i} \rho_{i} \bmod I^{5} F
$$

with $a_{i} \in F, \rho_{i} \in W(F(\psi) / F) \cap P_{4}(F)$ and $n$ minimal. Suppose $n>1$. Since $W(F(\psi) / F)$ is a strong 4-Pfister ideal, $\rho_{1}$ and $\rho_{2}$ are linked. Thus there is an $a_{n+1} \in \dot{F}$ and $\rho_{n+1} \in W(F(\psi) / F) \cap P_{4}(F)$ such that 
$a_{2}\left(\rho_{2} \perp-\rho_{1}\right)=a_{n+1} \rho_{n+1}$. We have:

$$
\begin{array}{rlrl}
q & \equiv a_{1} \rho_{1} \perp a_{2} \rho_{1} \perp-a_{2} \rho_{1} \perp a_{2} \rho_{2} \perp \frac{\perp}{i=3} a_{i} \rho_{i} & \bmod I^{5} F \\
& \equiv\left\langle a_{1}, a_{2}\right\rangle \rho_{1} \perp a_{n+1} \rho_{n+1} \perp \frac{\perp_{i=3}^{n} a_{i} \rho_{i}}{n+1} & & \bmod I^{5} F \\
& \equiv \sum_{i=3}^{n+1} a_{i} \rho_{i} & & \bmod I^{5} F
\end{array}
$$

This contradicts the minimality of $n$ and proves (b) for this case.

Case 2. $\psi$ is a Pfister neighbor:

Let $\psi$ be a neighbor to the (3-fold) Pfister form $\sigma$. Then $q \simeq \sigma \otimes$ $\left\langle b_{1}, \ldots, b_{m}\right\rangle$ by $[5,1.4]$. To prove (a), we need only show $m$ is even. Suppose $m$ is odd. Since $q \otimes F(\varphi)=0,(\sigma \otimes F(\varphi)) \otimes\left(\left\langle b_{1}, \ldots, b_{m}\right\rangle \otimes\right.$ $F(\varphi))=0$. If $\sigma \otimes F(\varphi) \neq 0$, then $\left\langle b_{1}, \ldots, b_{m}\right\rangle \otimes F(\varphi)$ is an odd dimensional zero advisor, which is impossible ([15, VIII 6.7]). Thus $\sigma \otimes F(\varphi)=$ 0 . Since $\operatorname{deg} \sigma=3$ and $\operatorname{dim} \varphi>5$, the Cassels-Pfister theorem implies $\varphi$ is a neighbor to $\sigma$, contrary to hypothesis. Thus $m$ is even and (a) holds.

Now write $\left\langle b_{1}, \ldots, b_{m}\right\rangle \equiv\langle 1, x\rangle \bmod I^{2} F$ for some $x \in \dot{F}$. Then $q \equiv\langle 1, x\rangle \sigma \bmod I^{5} F$ as desired.

COROLlaRY 3.3. If $F$ is 5-linked then for all conservative $\varphi$ over $F$, $W(F(\varphi) / F)$ is a Pfister ideal.

Proof. Let $q \in W(F(\varphi) / F)$; we may write $q=a_{1} \rho_{1} \perp q_{1}$ with $a_{1} \in \dot{F}$, $\rho_{1} \in W(F(\varphi) / F) \cap P_{4}(F)$ and $q_{1} \in W(F(\varphi) / F) \cap I^{5} F$, by (3.2). By [10, 5.1], $W(F(\varphi) / F) \cap I^{5} F$ is a Pfister ideal, hence $q_{1}$, and $q$, lie in $W(F(\varphi) / F)_{P f}$.

COROLlaRY 3.4. Suppose $\varphi$ is a conservative form over $F$ that is not embeddable. Then $W(F(\varphi) / F) \subset I^{5} F$.

Proof. Clearly $\varphi$ is not a Pfister neighbor, and $\operatorname{dim} \varphi \geq 7$ by (3.1). The result then follows from (3.2) since $W(F(\varphi) / F) \cap P_{4}(F)=0$.

In [9] it was shown that if $q \in W(F(\varphi) / F)$ then $2^{n} q \in W(F(\varphi) / F)_{P f}$, where $n=\operatorname{dim} q$. Thus if $\varphi$ is conservative but not embeddable then $W(F(\varphi) / F) \subset W_{t} F$ (see also [12]). Hence we have:

COROllary 3.5. Suppose $I^{5} F$ is torsion-free. Then a form $\varphi$ over $F$ is conservative if and only if it is embeddable. 
In particular, if $\operatorname{tr}$. d. $_{\mathbf{R}}(F) \leq 4$, then $\varphi$ is conservative if and only if it is embeddable.

CoROllary 3.6. Suppose $\varphi$ is a conservative form over $F$ that is not embeddable. If $q \in W(F(\varphi) / F)$ is non-zero, then $\operatorname{dim} q \geq 48$.

In particular, if $u(F)<48$, then a form over $F$ is conservative if and only if it is embeddable.

Proof. We may assume $q$ is anisotropic. By (3.4), $q \in I^{5} F$ and so by the Arason-Pfister Hauptsatz ([2]), $\operatorname{dim} q \geq 32$. If $\operatorname{dim} q=32$, then $q \in$ $G P(F)$ and $\varphi$ is embeddable; thus $\operatorname{dim} q>32$. By (3.2), $16 \mid \operatorname{dim} q$, so $\operatorname{dim} q \geq 48$.

4. Witt kernels over fields of finite Hasse number. As was done in [11], for an anisotropic form $q$ we define $N(q)$ to be $\operatorname{dim} q-q^{\operatorname{deg} q}$.

LEMMA 4.1. Suppose $\varphi \notin G P(F)$ and $q$ is an anisotropic form with $q \in W(F(\varphi) / F)$. Then,

(i) $2^{\operatorname{deg} q}>\operatorname{dim} \varphi$;

(ii) if $N(q)<2 \cdot \operatorname{dim} \varphi$ then $q \in G P(F)$.

Proof. (i) follows from [12, Prop. 13] and (ii) follows from [11, 1.6].

REMARK. A stronger inequality than (i) is shown in [12], namely that $2^{\operatorname{deg} q} \geq \operatorname{dim} \varphi+2^{\operatorname{deg} \varphi}$. It would be interesting to know if this can be improved to $2^{\operatorname{deg} q} \geq 2 \cdot \operatorname{dim} \varphi$ for non-Pfister neighbors $\varphi$. Note that if there exists a $q \in W(F(\varphi) / F)$ such that $2 \operatorname{dim} \varphi \geq 2^{\operatorname{deg} q}$ and $\varphi$ is not a Pfister neighbor then $W(F(\varphi) / F)$ is not a Pfister ideal. Namely, suppose $q=\perp_{i=1}^{n} x_{i} \rho_{i}$ with $\rho_{i} \in W(F(\varphi) / F) \cap P(F)$. Then for some $i, \operatorname{deg} \rho_{i} \leq$ $\operatorname{deg} q$ and the Cassels-Pfister theorem then implies $\varphi$ is a Pfister neighbor.

We next recall a definition due to Knebusch, Rosenberg and Ware (cf. $[14,1.2])$ which will be used frequently in this section:

Definition. We say $F$ satisfies the Strong Approximation Property (SAP) if for every clopen $S \subset X_{F}$ there exists an $e \in \dot{F}$ such that $e>0$ on $S$ and $e<0$ outside of $S$.

The following lemma is well-known.

LeMMA 4.2. If $\tilde{u}(F) \leq 2^{n}$, then $F$ is $n$-linked. In particular, $F$ is SAP. 
Proof. Let $\rho_{1}, \rho_{2} \in P_{n}(F)$. Then for any ordering $\alpha$ on $F$,

$$
\left|\operatorname{sgn}_{\alpha}\left(\rho_{1} \perp-\rho_{2}\right)\right|=\operatorname{dim} \rho_{1} \text { or } 0 \text {. }
$$

In particular, $\rho_{1} \perp-\rho_{2}$ is indefinite. Hence $\operatorname{dim}\left(\operatorname{ker}\left(\rho_{1} \perp-\rho_{2}\right)\right) \leq 2^{n}$ and the Witt index $i\left(\rho_{1} \perp-\rho_{2}\right) \geq 2^{n-1}$. Then, $\rho_{1}$ and $\rho_{2}$ are linked, by [5, 4.4].

For the second statement, $F$ is $n$-linked, so stably linked (cf. [6]) and hence $F$ is SAP by $[6,3.5]$.

LEMMA 4.3. Let $q \in W(F(\varphi) / F)$. If $\varphi$ is indefinite at $\alpha \in X_{F}$, then $\operatorname{sgn}_{\alpha} q=0$.

Proof. Since $\varphi$ is indefinite at $\alpha, \alpha$ extends to $F(\varphi)([9,3.5])$. Since $q \otimes F(\varphi)=0, \operatorname{sgn}_{\alpha} q=0$.

Proposition 4.4. Suppose $u(F) \leq 2^{n}$, and $\varphi$ is a conservative indefinite form over $F$. Then:

(i) If $2^{\dot{n}-1}<\operatorname{dim} \varphi \leq 2^{n}$, then $\varphi$ is a Pfister neighbor. In particular, $W(F(\varphi) / F)$ is a strong $n$-Pfister ideal.

(ii) If $2^{n-2}<\operatorname{dim} \varphi \leq 2^{n-1}$, then either:

(a) $\varphi$ is a Pfister neighbor and $W(F(\varphi) / F)$ is a strong $(n-1)$-Pfister ideal, or

(b) $\varphi$ is not a Pfister neighbor and every non-zero anistropic $q \in$ $W(F(\varphi) / F)$ is in $G P_{n}(F)$. In particular, $W(F(\varphi) / F)$ is a strong $n$-Pfister ideal.

Proof. Let $0 \neq q \in W(F(\varphi) / F)$ be anistropic. By (4.3) $\operatorname{sgn}_{\alpha} q=0$ for all $\alpha \in X_{F}$, so by Pfister's Local-Global Principle $q$ is torsion. Thus $\operatorname{dim} q \leq 2^{n}$.

(i) Here $\operatorname{dim} q<2 \operatorname{dim} \varphi$ and so $q \in G P_{n}(F)$ by (4.1). In particular, $\varphi$ is a Pfister neighbor.

(ii) Part (a) is known so suppose $\varphi$ is not a Pfister neighbor. By (4.1), $2^{\operatorname{deg} q}>\operatorname{dim} \varphi>2^{n-2}$ thus $\operatorname{deg} q \geq n-1$ and $N(q) \leq 2^{n}-2^{n-1}<$ $2 \operatorname{dim} \varphi$. (4.1) then implies $q \in G P(F)$. If $\operatorname{deg} q=n-1$, then $\varphi$ is a Pfister neighbor, contrary to the assumption of (b). Hence $q \in G P_{n}(F)$ and $W(F(\varphi) / F)$ is a strong $n$-Pfister ideal.

Both the statement and the proof of the following lemma are similar to the Pfister neighbor criterion of Elman, Lam and Wadsworth [8, 4.6]:

LEMMA 4.5. Let $F$ be formally real with $\tilde{u}(F) \leq 2^{n}$. Let $\varphi$ be a form over $F$, definite at some $\alpha \in X_{F}$, with $1 \in D(\varphi)$ and $\operatorname{dim} \varphi>2^{n-2}$.

(i) Let $m$ be the least integer such that $n \leq m$ and $\operatorname{dim} \varphi \leq 2^{m}$. Let $S$ be 
a non-empty clopen subset of $X_{F}$ such that $S \subset\{\alpha \mid \varphi$ is (positive) definite at $\alpha\}$. Then there exists $\rho \in W(F(\varphi) / F) \cap P_{m+1}(F)$ such that $\rho$ is definite at $\alpha$ iff $\alpha \in S$.

(ii) If $\operatorname{dim} \varphi=2^{m}+1, m \geq n$, then $\varphi$ is a Pfister neighbor.

Proof. In part (ii) let $S=\{\alpha \mid \varphi$ is definite at $\alpha\} . S$ is clopen since $S=\hat{\varphi}^{-1}(\{\operatorname{dim} \varphi\})$, where $\hat{\varphi}: X_{F} \rightarrow Z$ is the continuous function $\alpha \mapsto$ $\operatorname{sgn}_{\alpha}(\varphi)$.

For both parts (i) and (ii) there is an $e \in \dot{F}$ such that $e>_{\alpha} 0$ iff $\alpha \in S$, since $F$ is SAP. Set $\rho=2^{m}\langle 1, e\rangle$. For $\alpha \in X_{F}$ then:

$$
\operatorname{sgn}_{\alpha}(\rho \perp-\varphi)= \begin{cases}-\operatorname{sgn}_{\alpha} \varphi, & \text { if } e<_{\alpha} 0 \\ \operatorname{dim} \rho-\operatorname{dim} \varphi & \text { if } e>_{\alpha} 0 .\end{cases}
$$

In (i), $\left|\operatorname{sgn}_{\alpha} \varphi\right| \leq \operatorname{dim} \varphi \leq 2^{m} \leq \operatorname{dim} \rho-\operatorname{dim} \varphi$. In (ii), if $e<_{\alpha} 0,\left|\operatorname{sgn}_{\alpha} \varphi\right|$ $\leq \operatorname{dim} \varphi-2=2^{m}-1=\operatorname{dim} \rho-\operatorname{dim} \varphi$. Thus in both cases

$$
\left|\operatorname{sgn}_{\alpha}(\rho \perp-\varphi)\right| \leq \operatorname{dim} \rho-\operatorname{dim} \varphi, \text { for all } \alpha \in X_{F} .
$$

Set $\psi=\operatorname{ker}(\rho \perp-\varphi)$.

Suppose $\operatorname{dim} \psi>\operatorname{dim} \rho-\operatorname{dim} \varphi$. Then $\psi$ is indefinite. In (i), this forces $\operatorname{dim} \psi \leq 2^{n} \leq 2^{m} \leq \operatorname{dim} \rho-\operatorname{dim} \varphi$, and in (ii), since $\operatorname{dim} \psi$ is odd, $\operatorname{dim} \psi \leq 2^{n}-1 \leq \operatorname{dim} \rho-\operatorname{dim} \varphi$. In both cases we get a contradiction.

So $\operatorname{dim} \psi \leq \operatorname{dim} \rho-\operatorname{dim} \varphi$. In particular, the Witt index $i(\rho \perp-\varphi) \geq$ $\operatorname{dim} \varphi$. Thus $\varphi$ is a subform of $\rho$.

THEOREM 4.6. Suppose $\tilde{u}(F) \leq 2^{n}$ and $\varphi$ is a conservative form over $F$. If $2^{m-1}<\operatorname{dim} \varphi \leq 2^{n}$, with $m \geq n$, then either:

(i) $\varphi$ is a Pfister neighbor and $W(F(\varphi) / F)$ is a strong $m$-Pfister ideal, or

(ii) $\varphi$ is not a Pfister neighbor and $W(F(\varphi) / F)$ is a strong $(m+1)$ Pfister ideal.

Proof. We may assume $1 \in D(\varphi)$. We may also assume $\varphi$ is not indefinite and, in particular, that $F$ is formally real, by (4.4). Case (i) is known so assume $\varphi$ is not a Pfister neighbor.

Let $0 \neq q \in W(F(\varphi) / F)$ be anisotropic. We will show $q$ is isometric to a sum of multiples of $(m+1)$-fold Pfister forms in $W(F(\varphi) / F)$ by induction on $\operatorname{dim} q$.

Case 1. $\operatorname{dim} q \leq 2^{m+1}$ :

By (4.1), $2^{\operatorname{deg} q}>\operatorname{dim} \varphi>2^{m-1}$. So $\operatorname{deg} q \geq m$ and $N(q) \leq 2^{m+1}-2^{m}$ $=2^{m}<2 \operatorname{dim} \varphi$. This implies $q \in G P(F)$ by (4.1). If $\operatorname{deg} q=m$, then $\varphi$ is 
a Pfister neighbor, contrary to our assumption. Thus deg $q \geq m+1$. Since $\operatorname{dim} q \leq 2^{m+1}$ we obtain $q \in G P_{m+1}(F)$.

Case 2. $\operatorname{dim} q>2^{m+1}$ :

Set $S_{1}=\left\{\alpha \in X_{F} \mid \operatorname{sgn}_{\alpha} q \neq 0\right\}$ and $S_{2}=\left\{\alpha \in S_{1} \mid \operatorname{sgn}_{\alpha} q>0\right\}$. Both $S_{1}$ and $S_{2}$ are clopen, $S_{1}$ is non-empty (as $\operatorname{dim} q>\tilde{u}(F)$ ) and $S_{1} \subset\{\alpha \mid \varphi$ is (positive) definite at $\alpha$ \} by (4.3). Thus there is an $e_{2}$ such that $e_{2}>_{\alpha} 0$ iff $\alpha \in S_{2}$, since $F$ is SAP (set $e_{2}=-1$ if $S_{2} \neq \varnothing$ ), and a $\rho \in W(F(\varphi) / F) \cap$ $P_{m+1}(F)$ such that $\rho$ is definite at $\alpha$ iff $\alpha \in S_{1}$, by (4.5).

Set $q_{1}=\operatorname{ker}\left(e_{2} q \perp-\rho\right)$. Let $\alpha \in X_{F}$. Then:

$$
\operatorname{sgn}_{\alpha} q_{1}= \begin{cases}0, & \text { if } \alpha \notin S_{1}, \\ -\operatorname{sgn}_{\alpha} q-\operatorname{dim} \rho, \operatorname{sgn}_{\alpha} q<0, & \text { if } \alpha \in S_{1}-S_{2}, \\ \operatorname{sgn}_{\alpha} q-\operatorname{dim} \rho, \operatorname{sgn}_{\alpha} q>0, & \text { if } \alpha \in S_{2} .\end{cases}
$$

Thus for each $\alpha \in X_{F}, 2-\operatorname{dim} \rho \leq \operatorname{sgn}_{\alpha} q_{1} \leq \operatorname{dim} q-\operatorname{dim} \rho$ that is:

$$
\left|\operatorname{sgn}_{\alpha} q_{1}\right| \leq \max \left\{\operatorname{dim} q-2^{m+1}, 2^{m+1}-2\right\} .
$$

Thus, since $\tilde{u}(F) \leq 2^{n}$,

$$
\operatorname{dim} q_{1} \leq \max \left\{\operatorname{dim} q-2^{m+1}, 2^{m+1}-2,2^{n}\right\} .
$$

Now since $q, \rho \in W(F(\varphi) / F), q_{1} \in W(F(\varphi) / F)$. Applying the argument in Case 1 to $q_{1}$ (instead of $q$ ) we see that $\operatorname{dim} q_{1} \geq 2^{m+1}>2^{n}$. Hence, the largest term on the right in $\left(^{*}\right)$ must be $\operatorname{dim} q-2^{m+1}$. So $\operatorname{dim} q_{1} \leq \operatorname{dim} q-2^{m+1}$.

Since $q_{1}=e_{2} q \perp-\rho, \operatorname{dim} q_{1} \geq \operatorname{dim} q-\operatorname{dim} \rho=\operatorname{dim} q-2^{m+1}$. So $\operatorname{dim} q_{1}=\operatorname{dim} q-2^{m+1}, e_{2} q \simeq \rho \perp q_{1}$ and $q \simeq e_{2} \rho \perp e_{2} q_{1}$. Lastly, $e_{2} q_{1} \in$ $W(F(\varphi) / F)$ and $\operatorname{dim} q_{1}<\operatorname{dim} q$, so we are done by induction.

Remark. Case (ii) of Theorem 4.6 can occur. Consider $\varphi=$ $\langle 1,1,1,1,1,7\rangle$ over $F=\mathbf{Q}$. Since $\varphi$ is not indefinite, $\varphi$ is conservative namely $\langle\langle 1,1,1,1,1,7\rangle\rangle \in W(F(\varphi) / F)$. If $\varphi$ were a Pfister neighbor of some $\rho \in P(F)$, then since $5\langle 1\rangle<\varphi<\rho, \rho \simeq 8 \cdot\langle 1\rangle$ ([5, 2.7]). Thus $7 \in D(\langle 1,1,1\rangle)$, a contradiction. Hence $\varphi$ is a conservative non-Pfister neighbor while $\tilde{u}(F)=4$ and $\operatorname{dim} \varphi=6$. However we do have:

Proposition 4.7. Suppose $\tilde{u}(F) \leq 2^{n}$ and $\varphi$ is an anisotropic form over F. Then:

(i) If $\operatorname{dim} \varphi=2^{m}+1, m \geq n$, then $\varphi$ is a Pfister neighbor.

(ii) If $\operatorname{dim} \varphi=2^{m}, m \geq n$ and $\varphi$ is not indefinite, then $\varphi$ is a conjugate neighbor. 
Proof. We may assume $1 \in D(\varphi)$. Only (ii) is new and here $\varphi \perp\langle 1\rangle$ is anisotropic since $\varphi$ is not indefinite. Part (i) implies $\varphi \perp\langle 1\rangle$ is a Pfister neighbor, and hence $\varphi$ is a conjugate neighbor.

We now consider the forms $\varphi$ over $F$ with $\tilde{u}(F) \leq 2^{n}$ and $2^{n-2}<$ $\operatorname{dim} \varphi \leq 2^{n-1}$. This requires two lemmas, the first of which is well-known:

LEMMA 4.8. If $\tilde{u}(F) \leq 2^{n}$, then $J_{k} F=I^{k} F$ for $k \geq n$.

Proof. We may assume $F$ is real. Let $s=\operatorname{st}(F)$ be the reduced stability index as defined by Bröcker in [3]. SAP fields have $s=1$ ([7]) so:

$$
J_{k} F=I^{k} F+\left(J_{k} F\right)_{t}
$$

for each $k$ by [1, Lemma 2], where $\left(J_{k} F\right)_{t}$ denotes the torsion part of $J_{k} F$. Since $k \geq n,\left(J_{k} F\right)_{t} \subset I^{k} F$ and $J_{k} F=I^{k} F$.

Lemma 4.9. Suppose $\tilde{u}(F) \leq 2^{n}$ and $\varphi$ is an anisotropic form over $F$ with $2^{n-2}<\operatorname{dim} \varphi \leq 2^{n-1}$. Suppose also that there exists a $q \in W(F(\varphi) / F)$ of degree $n-1$. Then $\varphi$ is a Pfister neighbor.

Proof. We may assume $q$ is anisotropic, $1 \in D(q)$ and, by (4.4), that $\varphi$ is not indefinite. We induct on $\operatorname{dim} q$. If $\operatorname{dim} q \leq 2^{n}$, then $N(q) \leq 2^{n-}$ $2^{n-1}<2 \operatorname{dim} \varphi$. (4.1) then implies $q \in G P_{n-1}(F)$ and so $\varphi$ is a Pfister neighbor by the Cassels-Pfister theorem.

Now suppose $\operatorname{dim} q>2^{n} ; q$ is thus not indefinite. Set $S=\left\{\alpha \in X_{F} \mid q\right.$ is (positive) definite at $\alpha\} . S$ is non-empty and clopen in $X_{F}$. Using (4.3) and (4.5) we obtain a $\rho \in W(F(\varphi) / F) \cap P_{n+1}(F)$ such that $\rho$ is definite at $\rho$ iff $\alpha \in S$.

For $\alpha \in X_{F}$ :

$$
\operatorname{sgn}_{\alpha}(q \perp-\rho)= \begin{cases}\operatorname{dim} q-2^{n+1}, & \text { if } \alpha \in S \\ \operatorname{sgn}_{\alpha} q, & \text { if } \alpha \notin S .\end{cases}
$$

If $\operatorname{dim} q \leq 2^{n+1}$, then $\left|\operatorname{dim} q-2^{n+1}\right| \leq 2^{n}<\operatorname{dim} q$. If $\operatorname{dim} q>2^{n+1}$, then $\left|\operatorname{dim} q-2^{n+1}\right|<\operatorname{dim} q$. And if $\alpha \notin S$, then $\left|\operatorname{sgn}_{\alpha} q_{1}\right|<\operatorname{dim} q$ for all $\alpha \in X_{F}$.

If $\operatorname{dim} q_{1} \geq \operatorname{dim} q$, then $q_{1}$ is indefinite and of dimension greater than $2^{n}$, which is impossible. So $\operatorname{dim} q_{1}<\operatorname{dim} q$. By $[13,6.4], q=\rho \perp q_{1}$ implies $\operatorname{deg} q_{1}=n-1$. Thus by induction $\varphi$ is a Pfister neighbor.

REMARK. Lemma 4.9 says the first inequality of (4.1) can be strengthed to $2 \operatorname{dim} \varphi \leq 2^{\operatorname{deg} q}$ for non-Pfister neighbors $\varphi$ provided $\operatorname{dim} \varphi>2^{n-2}$ and $\tilde{u}(F) \leq 2^{n}$. 
THEOREM 4.10. Suppose $\tilde{u}(F) \leq 2^{n}$ and $\varphi$ is a conservative form over $F$. If $2^{n-2}<\operatorname{dim} \varphi \leq 2^{n-1}$, then either:

(i) $\varphi$ is a Pfister neighbor and $W(F(\varphi) / F)$ is a strong $(n-1)$-Pfister ideal,

(ii) $\varphi$ is not a Pfister neighbor and $W(F(\varphi) / F)$ is $a\{n, n+1\}$-Pfister ideal.

Proof. (i) is known so we may assume $\varphi$ is not a Pfister neighbor. Let $0 \neq q \in W(F(\varphi) / F)$ be anisotropic. Then by (4.1), $2^{\operatorname{deg} q}>\operatorname{dim} \varphi>2^{n-2}$ and so $\operatorname{deg} q \geq n-1$. By (4.9) $\operatorname{deg} q \geq n$, and so $q \in I^{n} F$ by (4.8). Thus $W(F(\varphi) / F) \subset I^{n} F$. Since $F$ is $n$-linked $[\mathbf{1 0}, 5.1]$ implies $W(F(\varphi) / F)$ is a $N$-Pfister ideal, where $N=\{n, n+1, \ldots\}$.

To finish then, we need only show any form in $W(F(\varphi) / F) \cap P_{t}(F)$, with $i \geq n+2$, is divisible by a form in $W(F(\varphi) / F) \cap P_{n+1}(F)$. Let $\sigma \in W(F(\varphi) / F) \cap P_{l}(F)$ with $i \geq n+2$. We may assume $\varphi$ is not indefinite and, in particular, that $F$ is real, by (4.3). We may also assume $1 \in D(\varphi)$. Let $S=\left\{\alpha \in X_{F} \mid \varphi\right.$ is (positive) definite at $\left.\alpha\right\}$. $S$ is non-empty and clopen in $X_{F}$. There is then a $(n+1)=$ fold Pfister form $\rho \in$ $W(F(\varphi) / F)$ such that $\rho$ is definite at $\alpha$ iff $\alpha \in S$. Using (4.3) we see that for all $\alpha \in X_{F}$ :

$$
\operatorname{sgn}_{\alpha}(\sigma \perp-\rho)= \begin{cases}\operatorname{sgn}_{\alpha} \sigma-2^{n+1}, & \text { if } \alpha \in S \\ 0, & \text { if } \alpha \notin S .\end{cases}
$$

So $\left|\operatorname{sgn}_{\alpha}(\sigma \perp-\rho)\right| \leq \operatorname{dim} \sigma-\operatorname{dim} \rho$. For all $\alpha \in X_{F}$. Since $\operatorname{dim} \sigma-\operatorname{dim} \rho$ $>2^{n}, \operatorname{dim}(\operatorname{ker}(\sigma \perp-\rho)) \leq \operatorname{dim} \sigma-\operatorname{dim} \rho$. Thus $\rho<\sigma$ and $\rho \mid \sigma$ by $[5,2.7]$.

REMARK. The result of (4.4)(ii) for non-real fields is stronger than the corresponding result (4.10) for real fields, namely for real fields we no longer have that $W(F(\varphi) / F)$ is a strong Pfister ideal. To see why this occurs we observe that $W(F(\varphi) / F)$ is a strong $n$-Pfister ideal iff there exists a $\rho \in W(F(\varphi) / F) \cap P_{n}(F)$ such that $\operatorname{sgn}_{\alpha} \rho=0$ precisely when $\varphi$ is indefinite at $\alpha$. This condition holds trivially if $F$ is non-real (take $\left.\rho=2^{n-1}\langle 1,-1\rangle\right)$.

To verify the observation, we first note that by (4.2) and [10, 3.1], $W(F(\varphi) / F)$ is a strong $n$-Pfister ideal iff for each $\sigma \in W(F(\varphi) / F) \cap$ $P_{n+1}(F)$ there exists a $\rho \in W(F(\varphi) / F) \cap P_{n}(F)$ such that $\rho \mid \sigma$. Suppose $W(F(\varphi) / F)$ is a strong $n$-Pfister ideal. Then, since $F$ is SAP, we may find a $\sigma \in W(F(\varphi) / F) \cap P_{n+1}(F)$ such that $\sigma$ is definite at $\alpha$ iff $\varphi$ is. Let $\rho \in W(F(\varphi) / F) \cap P_{n}(F)$ be such that $\rho \mid \sigma$. Then $\operatorname{sgn}_{\alpha} \rho=0$ iff $\varphi$ is 
indefinite at $\alpha$. On the other hand, suppose we have such a $\rho \in$ $W(F(\varphi) / F) \cap P_{n}(F)$ and let $\sigma \in W(F(\varphi) / F) \cap P_{n+1}(F)$. By (4.3),

$$
\left\{\alpha \in X_{F} \mid \operatorname{sgn}_{\alpha} \rho=0\right\} \subset\left\{\alpha \in X_{F} \mid \operatorname{sgn}_{\alpha} \sigma=0\right\},
$$

so $\left|\operatorname{sgn}_{\alpha}(\sigma \perp-\rho)\right| \leq 2^{n}$ for each $\alpha \in X_{F}$ and $\rho \mid \sigma([5,2.7])$. Thus $W(F(\varphi) / F)$ is a strong $n$ Pfister ideal.

COROLlaRY 4.11. If $\tilde{u}(F) \leq 8$, then $W(F(\varphi) / F)$ is a strong $k$-Pfister ideal, for some $k$, for every conservative $\varphi$ over $F$. In particular, this holds for $C_{3}$ fields, global fields and fields of transcendence degree $\leq 1$ over $\mathbf{R}$.

Proof. The first statement follows from (1.5) and (4.6). For the second statement see [4].

Lastly we can imporve (3.3).

CoROllaRy 4.12. Let $\tilde{u}(F) \leq 32$ and $\varphi$ a conservative form over $F$ which is not a Pfister neighbor. Then $W(F(\varphi) / F)$ is a:

3-Pfister ideal

4-Pfister ideal

$\{4,5\}$-Pfister ideal

$\{4,5,6\}$-Pfister ideal if $\operatorname{dim} \varphi=7$ or 8

$\{5,6\}$-Pfister ideal

$(n+2)$-Pfister ideal if $\operatorname{dim} \varphi=4$

if $\operatorname{dim} \varphi=5$

if $\operatorname{dim} \varphi=6$

if $9 \leq \operatorname{dim} \varphi \leq 16$

if $2^{n}<\operatorname{dim} \varphi \leq 2^{n+1}, n \geq 4$.

Proof. All but (4) have been done previously, so assume $\operatorname{dim} \varphi=7$ or 8. The proof of (3.3) shows $W(F(\varphi) / F)$ is a $\{4,5, \ldots\}$-Pfister ideal, while the second paragraph of the proof of (4.10) shows $W(F(\varphi) / F)$ is a $\{4,5,6\}$-Pfister ideal.

Acknowledgement. The author would like to thank the referee for many suggestions improving the readability of the paper.

\section{REFERENCES}

[1] J. K. Arason and M. Kneubsch, Über die Grade quadratischer Formen, Math. Ann., 234 (1978), 167-192.

[2] J. K. Arason and A. Pfister, Beweis des Krullschen Durschschnittsatzes für den Wittring, Invent. Math., 12 (1971), 173-176.

[3] L. Bröcker, Zur Theorie der quadratischen Formen über formal reellen Körpern, Math. Ann., 210 (1974), 233-256. 
[4] R. Elman, Quadratic forms and the u-invariant, III Proceedings of quadratic form conference, 1976, Orzech, G. (Ed.); Queen's papers in pure and applied mathematics, Vol. 46, pp. 422-444, Queen's University, Kingston, Ontario, Canada, 1977.

[5] R. Elman and T. Y. Lam, Pfister forms and K-theory of fields, J. Algebra, 23 (1972), 181-213.

[6] Q Quadratic forms over formally real fields and pythagorean fields, Amer. J. Math., 94 (1972), 1155-1194.

[7] R. Elman, T. Y. Lam and A. Prestel, On some Hasse principles over formally real fields, Math. Zeit., 134 (1973), 291-301.

[8] R. Elman, T. Y. Lam and A. Wadsworth, Function fields of Pfister forms, Invent. Math., 51 (1979), 61-75.

[9] _ Orderings under field extensions, J. Reine Angew. Math., 306 (1979), 7-27.

[10] , Pfister ideals in Witt rings, Math. Ann., 245 (1979), 219-245.

[11] R. Fitzgerald, Function fields of quadratic forms, Math. Zeit., 178 (1981), 63-76.

[12] E. Gentile and D. Shapiro, Conservative quadratic forms, Math. Zeit., 163 (1978), 15-23.

[13] M. Knebusch, Generic splitting of quadratic forms, I, Proc. London Math. Soc., 33 (1976), 65-93; II. the same 34 (1977), 1-31.

[14] M. Knebusch, A Rosenberg and R. Ware, Structure of Witt rings, quotients of abelian group rings, and orderings of fields, Bull. Amer. Math. Soc., 77 (1971), 205-210.

[15] T. Y. Lam, The algebraic theory of Quadratic Forms, W. A. Benjamin, Reading, Mass. 1973.

[16] _ Ten lectures on quadratic forms over fields, Proceedings of quadratic forms conference, 1976, Orzech, G. (Ed.) Queen's papers in pure and applied mathematics, Vol. 46, pp. 1-102. Queen's University, Kingston, Ontario, Canada 1977.

Received January 28, 1982.

Dartmouth COLlege

HANOVER, NH 03755 


\section{PACIFIC JOURNAL OF MATHEMATICS}

EDITORS

Donald BABBITt (Managing Editor)

University of California

Los Angeles, CA 90024

Hugo Rossi

University of Utah

Salt Lake City, UT 84112

C. C. Moore and Arthur Ogus

University of California

Berkeley, CA 94720

\section{J. DugundiI}

Department of Mathematics

University of Southern California

Los Angeles, CA 90089-1113

R. Finn and H. SAMELSON

Stanford University

Stanford, CA 94305

\section{ASSOCIATE EDITORS}
R. ARENS
E. F. BECKENBACH
B. H. NEUMANN
F. WOLF
K. YOSHIDA (1906-1982)

\section{SUPPORTING INSTITUTIONS}

UNIVERSITY OF ARIZONA

UNIVERSITY OF BRITISH COLUMBIA

CALIFORNIA INSTITUTE OF TECHNOLOGY

UNIVERSITY OF CALIFORNIA

MONTANA STATE UNIVERSITY

UNIVERSITY OF NEVADA. RENO

NEW MEXICO STATE UNIVERSITY

OREGON STATE UNIVERSITY
UNIVERSITY OF OREGION

UNIVERSITY OF SOUTHERN CALIFORNIA

STANFORD UNIVERSITY

UNIVERSITY OF HAWAII

UNIVERSITY OF TOKYO

UNIVERSITY OF UTAH

WASHINGTON STATE UNIVERSITY

UNIVERSITY OF WASHINGTON 


\section{Pacific Journal of Mathematics}

Vol. 109, No. $1 \quad$ May, 1983

Donald George Babbitt and V. S. Varadarajan, Formal reduction theory of meromorphic differential equations: a group theoretic view $\ldots \ldots \ldots \ldots 1$

Jo-Ann Deborah Cohen, Norms on $F(X) \ldots \ldots \ldots \ldots \ldots \ldots \ldots \ldots \ldots \ldots$

Robert Fitzgerald, Witt kernels of function field extensions $\ldots \ldots \ldots \ldots \ldots 89$

Hervé Jacquet and Joseph Andrew Shalika, The Whittaker models of induced representations .............................. 107

Masakiti Kinukawa, Some generalizations of contraction theorems for

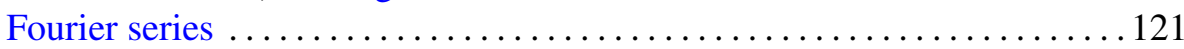

Joseph Weston Kitchen, Jr. and David A. Robbins, Sectional

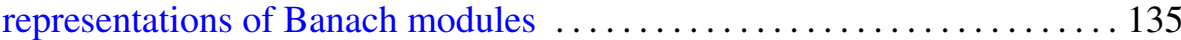

Victor Charles Pestien, Jr., Weak approximation of strategies in measurable gambling

Richard Scott Pierce and Charles Irvin Vinsonhaler, Realizing central

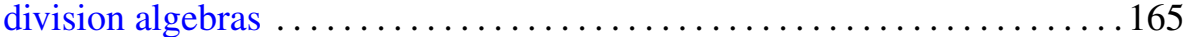

Walter Ricardo Ferrer Santos, Cohomology of comodules ............ 179

Marko Tadić, Harmonic analysis of spherical functions on reductive groups

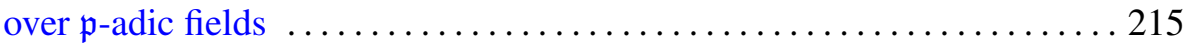

Lorenzo Traldi, The determinantal ideals of link modules. II . . . . . . . . 237

Alain J. Valette, A remark on the Kasparov groups $\operatorname{Ext}^{i}(A, B) \ldots \ldots \ldots 247$ 